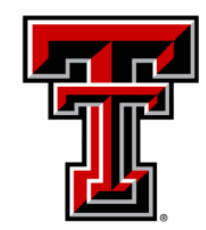

TEXAS TECH UNIVERSITY

Libraries"

\title{
PROBING CHANGES IN SECONDARY ELECTRON YIELD FROM COPPER ELECTRODES DUE TO SURFACE DEFECTS AND CHANGES IN CRYSTAL ORIENTATION
}

\section{The Texas Tech community has made this publication openly available. Please share how this access benefits you. Your story matters to us.}

\begin{tabular}{|c|c|}
\hline Citation & $\begin{array}{l}\text { H. Nguyen, M. Sanati, and R. P. Joshi, "Probing Changes in } \\
\text { Secondary Electron Yield from Copper Electrodes Due to Surface } \\
\text { Defects and Changes in Crystal Orientation," Journ. Appl. Phys. 126, } \\
123301 \text { (2019). https://doi.org/10.1063/1.5113642 }\end{array}$ \\
\hline Citable Link & https://hdl.handle.net/2346/86906 \\
\hline Terms of Use & $\begin{array}{l}\text { This article may be downloaded for personal use only. Any other } \\
\text { use requires prior permission of the author and AIP Publishing. }\end{array}$ \\
\hline
\end{tabular}




\section{Probing changes in secondary electron yield from copper electrodes due to surface defects and changes in crystal orientation}

Cite as: J. Appl. Phys. 126, 123301 (2019); https://doi.org/10.1063/1.5113642

Submitted: 05 June 2019 . Accepted: 02 September 2019 . Published Online: 25 September 2019

(D) H. K. A. Nguyen, M. Sanati, and (iD) R. P. Joshi
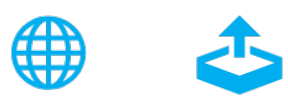

\section{ARTICLES YOU MAY BE INTERESTED IN}

Terahertz field confinement and enhancement in various sub-wavelength structures

Journal of Applied Physics 126, 120901 (2019); https://doi.org/10.1063/1.5110046

Reduction of capacitive coupling in inductively coupled plasmas by solenoid coils on dielectric window

Journal of Applied Physics 126, 123302 (2019); https://doi.org/10.1063/1.5116585

Investigation of plasmon-induced transparency and reflection in patterned graphene metamaterial

Journal of Applied Physics 126, 123101 (2019); https://doi.org/10.1063/1.5113669

Challenge us.

What are your needs for periodic signal detection?

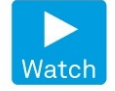

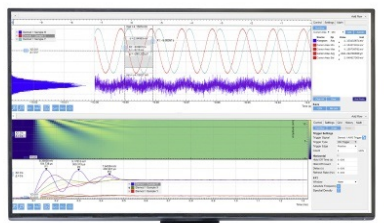

0
Zurich

- Instruments 


\title{
Probing changes in secondary electron yield from copper electrodes due to surface defects and changes in crystal orientation
}

Cite as: J. Appl. Phys. 126, 123301 (2019); doi: 10.1063/1.5113642

Submitted: 5 June 2019 - Accepted: 2 September 2019 .

Published Online: 25 September 2019

H. K. A. Nguyen, ${ }^{1}$ (D M. Sanati, ${ }^{2}$ and R. P. Joshi ${ }^{1, a)}$ (D)

\begin{abstract}
AFFILIATIONS
${ }^{1}$ Department of Electrical and Computer Engineering, Texas Tech University, Lubbock, Texas 79409, USA

${ }^{2}$ Department of Physics and Astronomy, Texas Tech University, Lubbock, Texas 79409, USA
\end{abstract}

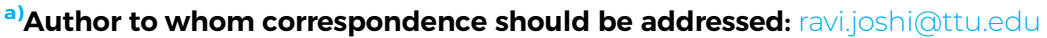

\begin{abstract}
There is considerable interest in mitigating secondary electron emission (SEE) from surfaces and electrodes produced by incident electrons, due to the deleterious effects of SEE in vacuum electron devices, accelerators, and other technologies. Since surface conditions are known to affect SEE, here the role played by crystal orientation and a vacancy (which is a simple example of a surface defect) is probed through Monte Carlo simulations. The effect of the lattice imperfection on the frequency-dependent permittivity, which then influences inelastic energy losses, mean free paths, and secondary generation profiles, is obtained on the basis of density-functional theory. The Monte Carlo simulations are in good agreement with previous experimental reports. The results indicate that the secondary electron yield for pure copper is the highest for the 110 orientation and the lowest for the 111 case, with a relatively higher differential predicted between a single vacancy and ideal copper for the 111 orientation. The results underscore the benefit of annealing or reducing inhomogeneities through laser or charged particle beam surface treatments.
\end{abstract}

Published under license by AIP Publishing. https://doi.org/10.1063/1.5113642

\section{INTRODUCTION}

Secondary electron emission (SEE) from dielectric and metal surfaces under bombardment of incident charged particles, especially electrons, is of concern and known to cause performance degradations in high power devices. Under suitable resonant near-vacuum conditions created by an input radiofrequency excitation, the electron population can increase in a continuous manner over time due to secondary electron emission from the electrode(s). This can then lead to effects such as strong wall charging, multipactor caused by multiplicative increases in secondary electron populations, curtailment of the efficiency and electric fields in RF cavities and amplifiers, ionization breakdown, and even initiation of electrostatic discharges on the outer surface of satellites leading to possible mission failure. ${ }^{1-18}$

SEE depends to a large extent on surface conditions ${ }^{19}$ and the material physics. For example, curtailing surface roughness through either laser irradiation or electron bombardment has been shown to reduce SEE. The inherent SEE physics depends on the material work function (WF) since it influences charge transfer at the surface. Electrons are naturally bound to a solid, and are prevented from escaping into the vacuum by the work function, which is an energy barrier between the Fermi energy and the vacuum level. ${ }^{20}$ Redistribution of charges at the surface arising from the "spillover" of electrons into the vacuum (e.g., via tunneling from the abrupt lattice terminations) modifies the potential step and thus influences WF. Also, differences linked to surface atomic arrangements can lead to shifts in the WF. As an example, the work function for tungsten has been measured to be $4.47 \mathrm{eV}$ and $5.25 \mathrm{eV}$ on atomically clean (111) and (110) surfaces, respectively. ${ }^{21} \mathrm{~A}$ thin film of polycrystalline gold exhibits a work function in the 4.4-4.7 eV range when exposed to ambient atmosphere. However, this value has been seen to increase to the $5.0-5.1 \mathrm{eV}$ range when cleaned in ultrahigh vacuum and increased even further to $5.3-5.4 \mathrm{eV}$ when sputter-cleaned under argon-ion bombardment. ${ }^{22}$

The essential point is that despite the same bulk material, the measured observables can vary due to differences in the surface 
characteristics, material purity, crystallographic orientation, surface roughness, and possible surface contamination. However, most reports in the literature ${ }^{16,23-29}$ have usually ignored these aspects. For example, the Vaughan model, ${ }^{30}$ and its modifications, ${ }^{31}$ are often used to describe SEE. However, the Vaughan approach is semiempirical, based on experimental observations, with no strong physical basis. It also does not incorporate any angular dependence of the incident electron ensemble. Even a more recent approach by Furman and Pivi ${ }^{32}$ does not take account of the work function, the role of crystal orientation, or defects.

A second aspect of the SEE and secondary electron yield (SEY) hinges on details of the trajectories of the incoming electrons, their energy-dependent interactions, and energy losses in the solid. These details dictate aspects such as the creation energy of the secondaries and their ability to escape the WF barrier. The detailed dynamics, including the energy losses and internal scattering, have been shown to depend on the dielectric properties of the target material. ${ }^{33}$ For example, the imaginary part of the permittivity influences the energy loss, and structural arrangements (including possible defects) influence the permittivity.

One of the current motivations here is to assess the role of crystal orientation on the SEY in copper material (taken as a representative solid target material) by calculating the secondary yield of electrons emerging from the (100), (110), and (111) facets. The focus is on understanding the underlying role played by changes in the work function, as well as variations in the energy loss functions associated with differences in permittivities. The influence of perturbations on the work function and frequency-dependent permittivity, created by a surface vacancy in copper at the three different orientations, is also evaluated. It is expected that changes due to surface vacancies would be small, given the relatively small fractional area that is affected, though the trends could be noteworthy. Variations in permittivity are probed by density-functional theory (DFT). This technique is also applied to recalculate the material parameters work function, and to assess effects of bond strength variations and the density of states (DOS), on the overall behavior. Finally, the results are used to generate predictive trends. For completeness, the role of electron-phonon interactions in comparison with energy losses to the plasmon modes is also discussed.

In terms of calculations, the role of material orientation and a simple surface vacancy on the SEY is first assessed by calculating the permittivities that influence the energy-dependent energy loss function (ELF). The metallic work function values are also obtained, and the data are collectively used to compute the electronic stopping power and energy loss rates. The latter form the basis for modeling the trajectories of incident electrons in copper based on a classical numerical Monte Carlo (MC) implementation based on Newtonian mechanics. Results of the secondary electron yield (SEY) are obtained for the [100], [110], and [111] orientations for copper. The MC scheme naturally builds in cascade effects, wherein secondary electrons can collide with other electrons within the material to produce subsequent tertiary electrons. This is an important effect, as first pointed out by Baroody. ${ }^{34}$ The cascade effect has been shown to lead a stronger generation of secondaries associated with a longer retention of electrons within the metal.

\section{METHOD DETAILS}

A physically accurate approach of obtaining values of the energy-dependent SEY is through numerical Monte Carlo simulations. ${ }^{35-37}$ This approach has been used for evaluation of SEY not just in metals, but in the context of semiconductor processing ${ }^{38}$ and physical processes in various solids, ${ }^{39-41}$ with different Monte Carlo strategies. ${ }^{42}$ It tracks the histories of both primary and secondary electrons through the target and accounts for the inelastic and elastic collisions between electrons and the host atoms. ${ }^{43-48}$ The screened Rutherford formula ${ }^{43}$ can be used for elastic scattering. On the other hand, inelastic processes involving excitations and ejections of atomic electrons, plasmon scattering, and phonons interactions lead to stronger energy dissipation but have a modest effect on changes in the electron direction. These inelastic processes have been treated using a continuous slowing down model based on Joy and Luo's generalization $^{44}$ of Bethe's stopping power formula. ${ }^{40,46,47}$ Improved formulations for inelastic scattering ${ }^{33,48}$ exist. For example, it has been shown that dielectric function modeling based on Penn's theory $^{33}$ yields the Bethe stopping powers. ${ }^{42,49}$ These calculated electron mean free paths (MFPs) also fit experimental data over a wide energy span ranging from several electron volts to several kilo-electron-volts for many elements and compounds. Thus, the use of Mott's elastic scattering cross section ${ }^{50}$ and Penn's dielectric function approach to electron inelastic scattering could be used for Monte Carlo simulations of SEY. The use of the dielectric function formalism has been accepted as a superior and more complete approach that provides a unified treatment of electron inelastic scattering and the associated secondary electron generation.

The dielectric function formalism also lends itself well to Monte Carlo implementations. A secondary electron is generated from the energy lost by a primary, after accounting for the ionization threshold. These secondaries can then go on to generate further tertiaries in a cascade process. In this manner, a Monte Carlo based simulation that includes the DFT analysis for the permittivity, mean free path, and the energy loss function, naturally folds in the spatiotemporal evolution of all electrons (i.e., primary, secondary, tertiary, etc.) throughout the sample. The ultimate demise of any particle is chosen to occur when their energy falls below the thermal value of $1.5 k_{B} T$, with $k_{B}$ being the Boltzmann constant and $T$ the absolute temperature. However, for this approach, accurate calculations of the complex frequencydependent dielectric function become essential.

Calculations of permittivity can be carried out based on DFT. ${ }^{51}$ Such rigorous electronic-structure methods have been successfully implemented to study the optical properties of metals. ${ }^{52-54}$ Employing such methods is beneficial, especially when examining variations on the nanoscale, and lead to a parameter-free bottom-up modeling. Here, electronic-structure calculations have been carried out using the Vienna $A b$ initio Simulation Package $(V A S P)$, a first-principles density-functional pseudopotential software tool. ${ }^{55-57}$ The VASP calculations use plane-wave basis sets and ultrasoft Vanderbilt-type pseudopotentials. ${ }^{58}$ The calculations were performed within the generalized gradient approximation (GGA) to the exchange-correlation potential as parameterized by 
Perdew-Burke-Ernzerhof. ${ }^{59}$ VASP also has the default setup for the Hellmann-Feynman forces. Furthermore, in the VASP code, the projector augmented-wave method ${ }^{60,61}$ was used with a cutoff kinetic energy of $345.9 \mathrm{eV}$. Besides $d$ electrons, only semicore $p$ electrons were considered as valence electrons. The crystal was represented, for the (100), (110), and (111) surfaces, by 343 atom periodic cells with 7 layers in each orthogonal direction. The electronic degrees of freedom were optimized with a conjugate gradient algorithm, and both cell constants and ionic positions (the upper two layers) are fully relaxed. A $3 \times 3 \times 1$ Monkhorst-Pack ${ }^{62}$ mesh was used to sample the Brillouin zone. Following Gajdoš et al., ${ }^{63}$ the frequency-dependent complex dielectric tensor was obtained for different surface orientations.

For the structures studied in this work, it was necessary to determine the optimum conditions required for the calculations, namely, the most reliable $k$-point mesh and energy cutoff. The energy cutoff controls the number of plane waves used in the basis set. In calculations based on plane-wave basis set, one should always test the convergence of the physical properties of the system (usually convergence of the energy for identical crystal structures). For periodic systems, the integrals are performed over the finite points (k-points) inside the Brillouin zone. Choosing sufficient number of $\mathbf{k}$-points is essential to ensure the convergence of results in any first-principles calculations. Firstly, the total energy was calculated using $\mathbf{k}$-point meshes of $3 \times 3 \times 2$ and $6 \times 6 \times 4$ resulting in less than $1 \mathrm{meV} /$ atom difference. The small energy difference allowed for the usage of a $3 \times 3 \times 2 \mathbf{k}$-point mesh for all remaining calculations in this work. Next, we calculated the effect of the energy cutoff on the total energy using cutoffs $300 \mathrm{eV}, 350 \mathrm{eV}$, and $400 \mathrm{eV}$. There was no significant change in calculated properties like work function (WF) and formation energy of vacancies, therefore, we used an energy cutoff of $400 \mathrm{eV}$ for all of the calculations presented in this work.

In first-principles calculations, it is essential to determine the overall optimized crystal structure, specifically the volume (and lattice constant). The equilibrium volume was determined by calculating the energy for several volumes and fitting the results to the Murnaghan equation of state. ${ }^{64,65}$ Calculated equilibrium lattice constants for the [100], [110], and [111] surface are $3.60 \AA$, $3.57 \AA$, and $3.61 \AA$, respectively. These values are slightly lower than the calculated Cu bulk lattice constant of $3.63 \AA .^{65}$

The formation energy of a defect is considered useful both for the determination of the existence (stability) of the defect and for the insight it gives to the strength of the interatomic bonding. The vacancy formation energy can be calculated from the following equation:

$$
\Delta H=E_{v a c}-\frac{N-1}{N} E_{c e l l}
$$

where $N$ is the total number of atoms in the cell and $E_{v a c}$ and $E_{\text {cell }}$ are the optimized energy of cell with and without vacancy, respectively.

Using the frequency-dependent permittivity [i.e., $\varepsilon(q, \omega)$ ] obtained from DFT calculations, the stopping power $(=d E / d R)$ for an electron of energy $E$, and the inverse inelastic MFP $\left(=\lambda^{-1}\right)$ could then be obtained. The specific relations connecting these quantities with $\varepsilon(q, \omega)$ are given as ${ }^{66,67}$

$$
\frac{d E}{d R}=\frac{\hbar}{\pi a_{0} E} \int_{0}^{\frac{E}{\hbar}} \hbar \omega d \omega \int_{q_{-}}^{q_{+}} \frac{\operatorname{Im}\left[\frac{-1}{\varepsilon(q, \omega)}\right]}{q} d q,
$$

where

$$
q_{ \pm}=\sqrt{\frac{2 m}{\hbar^{2}}}[\sqrt{E} \pm \sqrt{E-\hbar \omega}]
$$

and $a_{0}=\frac{4 \pi \varepsilon_{0} \hbar^{2}}{m_{e} e^{2}}=5.29177 \times 10^{-11} \mathrm{~m}$ is the Bohr radius. The inverse $\operatorname{MFP}^{m_{e} e^{2}}\left(=\lambda^{-1}\right)$ for inelastic collisions is given by

$$
\lambda^{-1}=\frac{\hbar}{\pi a_{0} E} \int_{0}^{\frac{E}{\hbar}} d \omega \int_{q_{-}}^{q_{+}} \frac{\operatorname{Im}\left[\frac{-1}{\varepsilon(q, \omega)}\right]}{q} d q .
$$

In the case when $\varepsilon(q, \omega)$ is independent of wavevector " $q$ " [i.e., $\varepsilon(q, \omega)=\varepsilon(\omega)]$, the stopping power and inverse inelastic MFP work out to

$$
\begin{gathered}
\frac{d E}{d R}=\frac{\hbar}{\pi a_{0} E} \int_{0}^{\frac{E}{\hbar}} \hbar \omega d \omega \frac{\varepsilon_{i m}(\omega)}{\varepsilon_{r}^{2}(\omega)+\varepsilon_{i m}^{2}(\omega)} \ln \frac{\sqrt{E}+\sqrt{E-\hbar \omega}}{\sqrt{E}-\sqrt{E-\hbar \omega}}, \\
\lambda^{-1}=\frac{\hbar}{\pi a_{0} E} \int_{0}^{\frac{E}{\hbar}} d \omega \frac{\varepsilon_{i m}(\omega)}{\varepsilon_{r}^{2}(\omega)+\varepsilon_{i m}^{2}(\omega)} \ln \frac{\sqrt{E}+\sqrt{E-\hbar \omega}}{\sqrt{E}-\sqrt{E-\hbar \omega}} .
\end{gathered}
$$

A wavevector independent approximation was used since the $q$-dependence is weak. This situation is crudely similar to treatments of the "electron-phonon" interactions for optical modes in solids, which are often calculated in the long-wavelength $(q \sim 0)$ limit. Very crudely, the wavevector dependence of the permittivity roughly has the following Lindhardt form: $\varepsilon(q) \sim \mathrm{A}+\mathrm{B} / q^{2}$. Hence, the integration over the wavevector then yields $\left\{\ln \left[\mathrm{A} q^{2}+\mathrm{B}\right]\right\} /(2 \mathrm{~A})$, which has to be evaluated at the two corresponding limits of $q_{\text {min }}$ and $q_{\max }$. In any case, the result is similar in form to the $\mathrm{q} \rightarrow 0$ case assumed here. Overall, trends in SEY are expected to roughly remain unaltered even with a full $q$-dependence, though the values might change somewhat and on-going detailed calculations will be reported elsewhere. ${ }^{68}$ For example, in a many-band calculation, the permittivity and hence the energy loss function, might not exhibit exactly the quadratic dependence on wavevector $q$, due to the different underlying physics of their origin.

In theory, energy carried by the incident electron swarm can also be transferred to the lattice through the electron-phonon interaction. For completeness, this aspect is briefly discussed and shown to be negligible in the present context of electrons hitting a target metal. The electron-phonon scattering rate $\tau_{e-p h}^{j}(q)$ for the $j$ th phonon mode (which could be either a Longitudinal Optical or Transverse Optical mode) is dependent on the phonon wavevector ${ }^{69}$ and given as, $\tau_{e-p h}^{j}(q)=\Xi^{2} m^{2} q /\left(2 \pi \hbar^{3} \rho_{M}\right)$, with $\Xi$ being the deformation potential, $q$ being the phonon wavevector, $m$ being the 
electron mass, and $\rho_{M}$ being the metal density. For a typical metal electrode (e.g., copper), one would have the following representative values: $\quad \Xi=3.77 \mathrm{eV}, \quad \rho_{M}=8.96 \times 10^{3} \mathrm{~kg} / \mathrm{m}^{3}, \quad \hbar \omega=24.8 \mathrm{meV}$, and $m=9.1 \times 10^{-31} \mathrm{~kg}$. A quick ballpark estimate for a phonon wavevector $q \sim 5 \times 10^{7} \mathrm{~m}^{-1}$ yields a rate of $2.275 \times 10^{8} \mathrm{~s}^{-1}$. For a representative $100 \mathrm{eV}$ electron, the value of the energy loss function (ELF) in energy per distance is then $\tau_{e-p h}^{j} \hbar \omega / v$, where the velocity $v$ would be related to the electron energy $E: v=(2 E / m)^{1 / 2}$. With typical phonon energies of $\sim 24.8 \mathrm{meV}$, this then yields an ELF of $\sim 1.5 \times 10^{-19} \mathrm{~J} / \mathrm{m}$. This estimate for the loss to the phonon bath is orders of magnitude lower than the ELF to plasmons, as shown later in Sec. III. Hence, the electron-phonon interaction can be neglected in the present context.

The motion of the electrons incident onto a copper target, with its subsequent scattering in the host, and possible creation of secondary electrons were simulated based on a kinetic Monte Carlo (MC) procedure. Details of such work in our group performed by our group have already been published elsewhere. ${ }^{70,71}$ In our implementation, when primary electrons enter the target material, a certain portion is backscattered elastically, while the remainder penetrates into the material. Some of these latter, then scatter from atoms inside the material and could be reflected back out. The scattering proceeds on the basis of the usual drift-and-scatter sequence, typical in a MC scheme. The path length $L_{i}$ for each drift component was obtained by weighting the energy-dependent mean free path $\lambda(E)$ as given in Eq. (3b), with a random number $r_{i}$ [i.e., $L_{i}=-\lambda(E) \ln \left(r_{i}\right)$ ]. The energy transfer $\Delta E$ was then taken to be $\Delta E=L_{i} \frac{d E}{d R}$, with the last term computed from Eq. (3a). Due to this transfer, the energy of the incoming electron was correspondingly reduced by $\Delta E$ to yield a new energy (and absolute velocity magnitude) for the primary particle. The usual assignment of new angles in the spherical coordinate system $(\theta$ and $\varphi)$ as discussed in the literature ${ }^{35-37}$ then yielded the new directional velocities for the primary upon effective deflection. Additionally, the energy loss $\Delta E$ less the ionization energy was assigned to the secondary electron, with the usual angular distribution being used to assign the velocity components. From the DOS results, the secondaries were predicted to emerge from the core locations. Thus, variable energies, angular direction, and velocity components for each particle were all included. Also, the trajectories of all the electrons in the system were tracked, i.e., the primaries, secondaries, tertiaries, and other subsequent offspring. The motion of an electron was deemed to be terminated once its energy fell to or below the thermal energy. Over time, some electrons emerge back out after successive scattering events, which yield the secondary emission coefficients from the ratio of the emergent population to the total incident number.

It was assumed that each inelastic collision produces a knock-on secondary electron by transferring $\Delta E$ to a shell electron. The primary electron of energy $E$ upon such an inelastic scattering event has an energy $E-\Delta E$, leaving the excited secondary electron with energy $\Delta E-E_{B}$, with $E_{B}$ an effective binding energy. In theory, the energy transfer from the primary could also occur from a Fermi electron. However, based on the density of states, we discounted this possibility. A result of the energy-dependent density of states (DOS) is given in Fig. 1. The plot shows the DOS vs energy obtained from DFT calculations in copper for the [100], [110], and [111] cases. The Fermi level $\left(E_{F}\right)$ is also shown, and a very low density is apparent in the neighborhood of $E_{F}$.

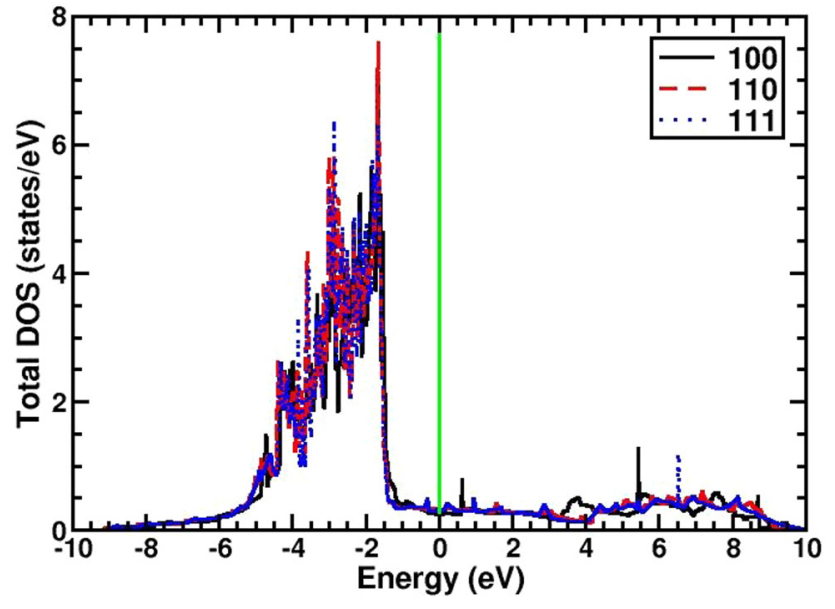

FIG. 1. Plot of the density of states (DOS) vs energy obtained from DFT calculations in copper for the [100], [110], and [111 directions. The Fermi level $\left(E_{F}\right)$ is also shown, and a very low density is apparent in the neighborhood of $E_{F}$

Finally, for the MC simulations carried out here, the cases of a surface vacancy in copper were treated by using a 49 atom periodic cell at the surface in the $x-y$ plane. Electrons were taken to be incident randomly over the area of the unit periodic cell. Electrons were chosen to be incident normally, though the technique is general and different angular distributions could have been easily assigned as well as reported elsewhere. ${ }^{72}$

\section{RESULTS AND DISCUSSION}

Calculated vacancy formation energies from the DFT simulations, along the [100], [110], and [111] directions were found to be $0.79 \mathrm{eV} /$ vacancy, $0.71 \mathrm{eV} /$ vacancy, and $1.00 \mathrm{eV} /$ vacancy, respectively. The $\mathrm{Cu} 111$ formation energy result is in fairly good agreement with the other first-principles calculated value of $0.92 \mathrm{eV} /$ vacancy. $^{73}$ The higher vacancy formation of the [111] surface with respect to [110] and [100] is an indication of a strong directional bonding between $\mathrm{Cu}$ atoms along the [111] direction. The directional bonding is directly related to the density of bonding electrons. Therefore, one expects that the work function of these surfaces will follow the same trend. That is, the work function of the [111] surface would be the highest, followed by that of the [100] surface, and the lowest for the [110] surface. Solely based on this aspect, though there are other considerations, might crudely expect the SEY to be the highest for 110 copper and the lowest for the 111 oriented crystal.

Calculated WF values for clean ideal surfaces, and surfaces with a vacancy, are given in Table I for the [100], [110], and [111] directions. For comparison, experimental data for a clean surface is also provided in the table. From the values in Table I, one can conclude that the calculated data are in very good agreement with experimental values, ${ }^{74}$ including the trend that the WF for the [111] case would be the highest and the [110] surface would be the lowest. 
TABLE I. Calculated work functions (in electron volts) of $\mathrm{Cu}$ for clean surfaces and surfaces with a vacancy along with available experimental measurements ${ }^{74}$ for the various surfaces.

\begin{tabular}{lccc}
\hline \hline $\begin{array}{l}\text { Surface WF } \\
(\mathrm{eV})\end{array}$ & $\begin{array}{c}\text { Clean } \\
\text { (this work) }\end{array}$ & $\begin{array}{c}\text { Vacancy } \\
\text { (this work) }\end{array}$ & $\begin{array}{c}\text { Clean } \\
\text { (exp.) } \pm 0.03\end{array}$ \\
\hline$[100]$ & 4.52 & 4.50 & 4.58 \\
{$[110]$} & 4.38 & 4.37 & 4.48 \\
{$[111]$} & 4.81 & 4.78 & 4.92 \\
\hline \hline
\end{tabular}

As one might expect, the creation of a vacancy slightly reduces the work function. This is due to the weakening of the bonds among the surrounding atoms and the donation of electron(s) to the $\mathrm{Cu}$ surface. The increased electron density at or near the Fermi level can reduce the work function of the system. To verify, the total density of states (TDOS) at the Fermi level for the various orientations were calculated, the results are given in Table II. From Table II, one can conclude that after the creation of a vacancy, the TDOS at the Fermi level for all the surfaces increases slightly, resulting in a small decrease in the work function. A comparison between Tables I and II allows one to see the direct relation between the TDOS and the work function, specifically for the clean surfaces and those with a vacancy. The results show the $\mathrm{Cu}$ [111] surface as having the highest change in TDOS (at the Fermi level) and work function value, while the $\mathrm{Cu}$ [110] surface has the lowest change between an ideal surface and that with a vacancy.

The response of the electronic density of a material to an electric field is measured by its polarizability. A weaker bond between atoms makes it easier for electronic shifts in charge density distribution, thus increasing the polarizability (permittivity) of the system. Hence, a vacancy can be expected to enhance permittivity, and hence dissipation in the system, leading to higher energy losses for incident electrons. Consequently, one expects the [100] and [110] surfaces to have lower permittivity values in comparison to the copper material along [111]. Also then, one would expect incident electrons to not penetrate as deep in the copper for the [111] case.

Results of the frequency-dependent permittivity for both an ideal copper lattice and one containing a surface defect, obtained from the DFT-based VASP tool, are shown in Fig. 2. Three different orientations (along [100], [110], and [111]) were used for the calculations. Figure 2(a) shows the magnitude of the real part of permittivity in copper, while Fig. 2(b) is the energy-dependent imaginary part. The peak around $2 \mathrm{eV}$ in Fig. 1(b) is indicative of inelastic energy losses in this energy range. The stopping power obtained from the above permittivity characteristic for pure copper

TABLE II. Total density of states (TDOS) at the Fermi level for different surfaces, including clean surfaces and those with a vacancy.

\begin{tabular}{lcc}
\hline \hline TDOS (states/eV atom) & Clean & Vacancy \\
\hline$[100]$ & 0.222 & 0.240 \\
{$[110]$} & 0.291 & 0.293 \\
{$[111]$} & 0.148 & 0.367 \\
\hline \hline
\end{tabular}
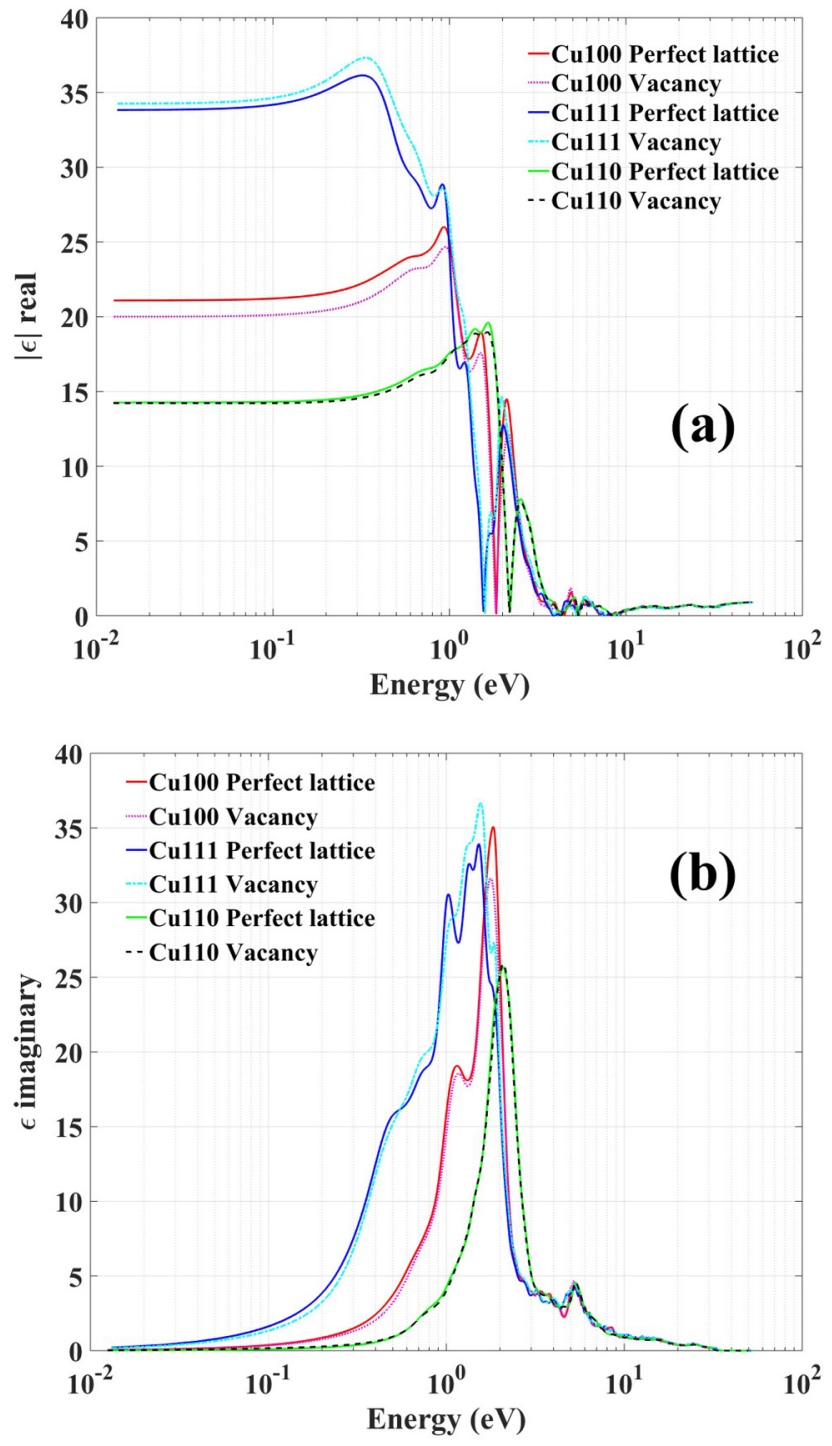

FIG. 2. Plot of the energy-dependent permittivity $(\varepsilon)$ magnitudes for copper obtained from VASP simulations under different conditions. A total of six cases were considered, the [100], [110], and [111] orientations for both ideal copper material and copper containing a single vacancy. (a) Magnitude of the real part of permittivities $\varepsilon_{R}(\omega)$ and (b) imaginary part $\varepsilon_{i m}(\omega)$ of the permittivity.

and with a single vacancy is shown in Fig. 3 for the [100], [110], and [111] orientations. The plot was obtained using Eq. (3a) with values of the real $\left[\varepsilon_{r}(\omega)\right]$ and imaginary $\left[\varepsilon_{\mathrm{im}}(\omega)\right]$ permittivities as a function of frequency obtained from the results shown in Fig. 2. The results for all six cases are fairly close to each other, exhibit a similar trend, and display a peak at around $40 \mathrm{eV}$. The stopping power for the 111 orientation is predicted to be the largest, for both ideal material and the single vacancy case. This signifies that the energy loss is higher, the primary electrons lose energy faster, 


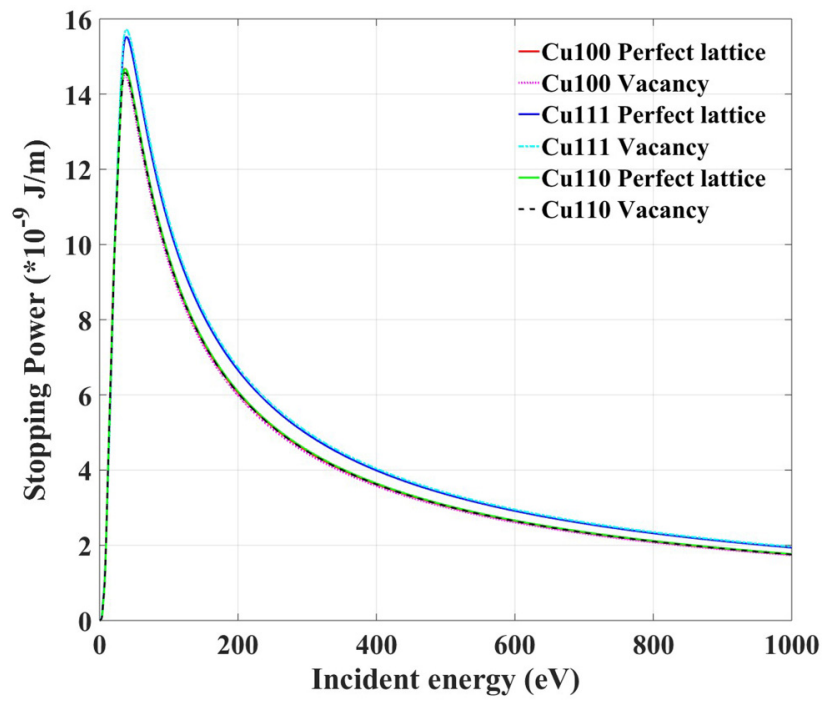

FIG. 3. The stopping power obtained from the permittivity characteristics of Fig. 2 for all six cases discussed. The [100] and [110] curves overlap to a large extent.

and hence, would typically lead to a lower number of secondary electron creation events starting from a given initial primary electron energy. However, the electrons in this case would be created closer to the surface. Finally, the inelastic mean free path $\lambda$ for defect-free copper as a function of electron energy for the [100],

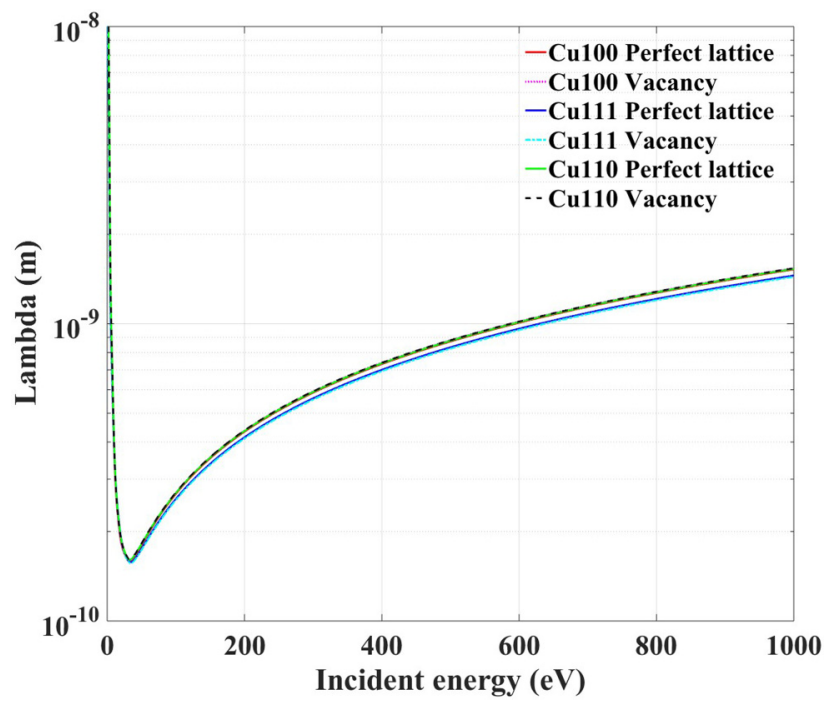

FIG. 4. The inelastic mean free path for defect-free copper as a function of electron energy for the [100], [110], and [111] orientations of copper in an ideal structure and with a single surface vacancy. The [100] and [110] curves overlap to a large extent.
[110], and [111] orientations of copper is shown in Fig. 4. The MFP is seen to range around a few nanometers, though at values around $40 \mathrm{eV}$ where the stopping power is high, the MFP is predicted to be much shorter, in the subnanometer range. At very low energies, the MFP could be much larger, at or beyond $50 \mathrm{~nm}$. Again, comparisons of the various values show the predicted MFPs to be the lowest for the [111] orientation.

Finally, simulations of incident electron transport within the copper were obtained based on the Monte Carlo approach. The stopping power, energy losses, and scattering angles were all included with a screened Rutherford formula for elastic scattering and the dielectric function formalism for the treatment of electron inelastic scattering and associated secondary electron generation. For greater insights and a more transparent analysis into the SEY results obtained, it is perhaps instructive to examine the energydependent total density of states (TDOS). The TDOS is indirectly a measure of the number of valence electrons and indicative of the number of initial sites for the formation of secondaries. The total density of states (TDOS) as a function of energy for the three crystal orientations of [100], [110], and [111] is shown in Fig. 5. For each case, the results obtained from DFT calculations are shown for an ideal defect-free crystal and a surface with one vacancy. Values for [111] copper are the lowest, followed by [100] copper, with the highest levels for the [110] material. Furthermore, the difference between ideal defect-free material and a surface with one vacancy is predicted to be marginal. Differences in TDOS for the [100] orientation are also fairly small, with the curve for the ideal material having a slight additional peak at about the $-1.1 \mathrm{eV}$ energy level. Finally, for the [110] orientation, the TDOS is the highest among all orientations considered. Furthermore, for this [110] case, the TDOS values are higher for ideal copper, especially at the low energies, as compared to the case with a vacancy.

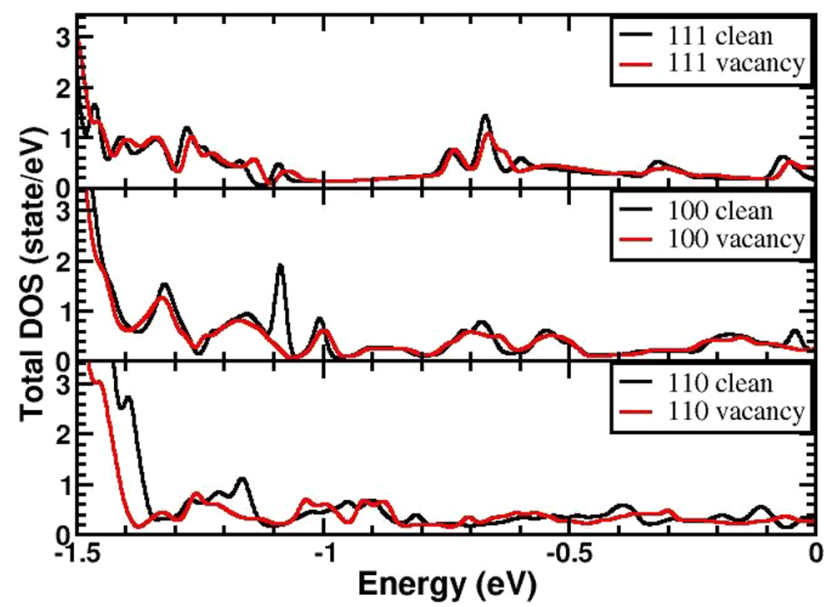

FIG. 5. The total density of states (TDOS) as a function of energy for the three crystal orientations of [100], [110], and [111]. For each case, the results obtained from DFT calculations are shown for an ideal defect-free crystal and a surface with one vacancy. These curves are zoomed in versions of the data shown in Fig. 1. 
Results obtained from MC simulations for SEY as a function of incident energy are shown in Fig. 6 . The data reported by Baglin et al., ${ }^{75}$ Ding et al., ${ }^{76}$ and Zhang et al. ${ }^{77}$ are also given for comparison. The data of Zhang et al. ${ }^{78}$ show the dynamic nature of the SEY and point to changes in the characteristics of the system over time. Their observations were recorded after subjecting the samples to argon-ion cleaning, which resulted in surface modification. For example, their results after a 40-min cleaning yielded a lower SEY than a 5-min treatment. This clearly demonstrates the role of surface treatment and modification on the SEY. This behavior is similar to that reported by Watts et al. ${ }^{19}$ The simulation results are seen to lie close to the Baglin ${ }^{75}$ data. Since statistical variations are inherent in the approach, each data point was obtained as an average of four different simulations, each with a different starting random number. The overall results for each case were quite close and within $5 \%$ of each other. The results indicate that the SEY for pure copper is the highest for the 110 orientation, and the lowest for the 111 case. This trend and sequence of relative magnitudes is qualitatively similar to the integrated results from the TDOS curves of Fig. 5. Furthermore, the integrated TDOS for both the [100] and [110] orientations is seen to be larger for the clean surface and less with a vacancy. Hence, with vacancy, one would correspondingly expect lower SEY values for the [110] and [100] cases, an outcome that is seen in the results of Fig. 6. The slight reductions in the SEY values for copper in the [110] and [100] cases is qualitatively in keeping with the report on the effects of gas bombardment. ${ }^{78}$ Presumably, the bombardment, which cased defect formation at or near the surface, was responsible for the SEY reduction. However, the use of a different material in Ref. 78 precludes direct quantitative comparisons. In other reports, Yang et al. ${ }^{79}$ found that argon-

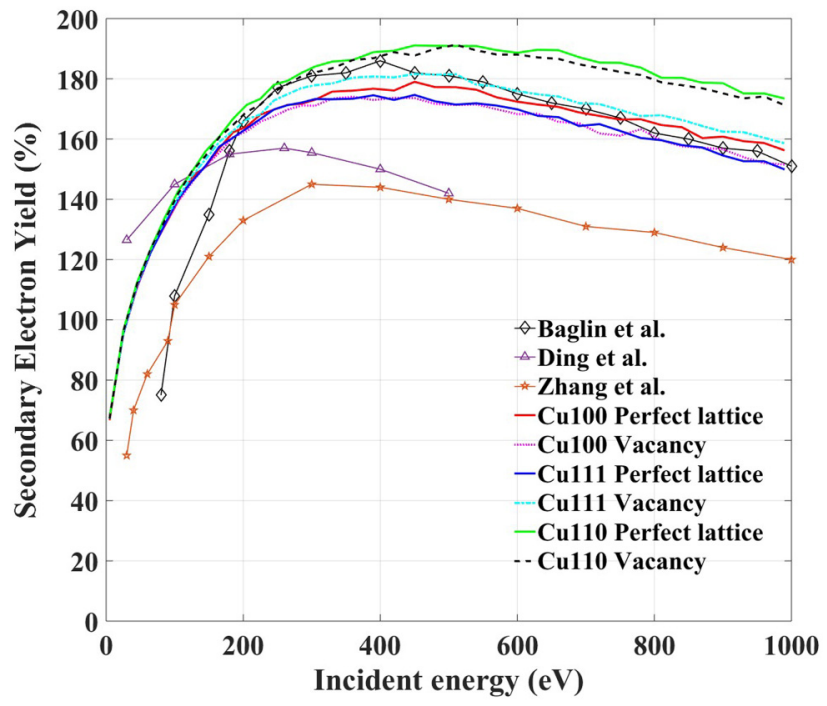

FIG. 6. Secondary electron yield vs electron energy obtained from Monte Carlo calculations for the six cases of a perfect copper lattice and a copper host containing a surface vacancy, along the [100], [110], and [111] directions. In addition, values reported by Baglin et al., ${ }^{75}$ Ding et al., ${ }^{76}$ and Zhang et al. ${ }^{77}$ are also given for comparison. ion sputtering reduced the SEY of gold by eliminating contamination and producing a rough surface due to ion bombardment. Furthermore, $\mathrm{Hu}$ et al. ${ }^{80}$ analyzed the influence of bombardment intensity and ion energy on the SEY of copper plates, and optimized ion-beam parameters were obtained for achieving the lowest SEY for copper. For the 111 orientation, the DOS was slightly less with a vacancy than for the defect-free material. Consequently, a slightly higher SEY is predicted with a vacancy as compared to the ideal surface in Fig. 6. Furthermore, for this orientation, the vacancy has a much lower work function than that of the perfect lattice. This again leads to a higher SEY with a vacancy over the defect-free case for 111 copper. If one looks at $-1.5 \mathrm{eV}$ energy region of TDOS curves of Fig. 5, the values for vacancy are seen to be shifted to the left and are lower for the [100] and [110] orientations. Consequently, one can expect more energy to be needed to extract the electrons. The shift is even higher for the [110] surface, and the outcome again is in agreement with the predicted SEY results.

Finally, a quick comment on the relatively higher differential in SEY between the case of a single vacancy over ideal copper, for the [111] orientation. This can qualitatively be understood from the energy formation standpoint. The [111] orientation was seen to have a high vacancy formation energy and, therefore, stronger bonding with more bonding electrons playing a role. In creating a vacancy since one has to break some bonds. Hence, more electrons can be expected to be released in going over from a defect-free material to a one with a vacancy. This relative change is not as large for the [110] and [100] surfaces, and correspondingly, neither are the differences in their SEY values, with and without a vacancy. In any event, it has been shown through the present simulations that a simple vacancy in the [111] direction can affect the SEY to some degree. It is thus conceivable that other defects (such as oxygen, hydrogen, etc.) in the material could also alter the SEY results. Hence, a natural progression would be to investigate the effects of other defects such as dislocations, grain boundaries, and dissolved gases. The latter would especially be dynamic in nature, with possible outgassing during the operation of such high power devices, and hence, fluctuations in SEY values.

Finally, it may be mentioned for completeness that a dependence of SEY on the incident angle would arise. ${ }^{81}$ In fact, this is a very interesting point, since a simple minded argument might lead to increased SEY for oblique incidence as the penetration depth can be smaller for this condition, leading to a larger "escape probability" for the secondaries. However, researchers have also reported the anomalous experimental behavior of the SEY not increasing with higher incident angle. ${ }^{82}$ Some of this was proposed as being the result of porosity at the surface. Besides, the use of roughened surfaces to induce greater reflection and scattering at the surface undulations have been proposed as a way to reduce SEY. Thus, the importance and relevance of the angular variability remains an important issue. For example, the geometrical morphology of sample surfaces was tailored to create triangular, rectangular, and microporous grooves through laser and chemical etching. This effectively resulted in angular variations experienced by the traversing electrons and led to SEY reductions. ${ }^{83}$ Also, on another cautionary note, the experimental data for copper shown here for comparison might have included grain boundaries or polycrystalline material. Though results 
for single crystal $\mathrm{Cu}[100]$ had been reported years ago, ${ }^{84}$ however, their data were not used here for comparison as the measurement techniques might not have been as sophisticated and the energy range was much lower, which precluded the attainment of a local SEY maxima.

\section{SUMMARIZING CONCLUSIONS}

There is considerable interest in mitigating SEE from surfaces and electrode materials due to their deleterious effects. For example, in space-borne equipment, secondary electrons can lead to multiplication, generate RF noise, alter impedance, create local heating, and cause hardware damage and electrostatic discharges on the outer surface of satellites. Suppression of SEY has relied on the use of surface treatment techniques or geometric modifications. ${ }^{83,85-88}$ However, since the surface condition/morphology is known to affect SEY, it becomes important to understand the material aspects and the role played by the surface. Here, the presence of simple defects, such as a vacancy, was probed through Monte Carlo simulations. The effect of the lattice imperfection on the frequency-dependent permittivity $\varepsilon(\omega)$ and hence the inelastic scattering, as well as deviations in work function, were included. Evaluations of $\varepsilon(\omega)$, with and without defects, were obtained on the basis of DFT.

The results indicate that the SEY for pure copper is the highest for the 110 orientation, and the lowest for the 111 case. This sequence of relative magnitudes is qualitatively in keeping with the trend exhibited by the density of states. For the [111] orientation, the DOS was slightly less with a vacancy than for the defect-free material. Consequently, a slightly higher SEY was predicted with a vacancy as compared to an ideal surface. Furthermore, for this orientation, the vacancy has a much lower work function than that of the perfect lattice, which also contributed to a higher SEY over the defect-free case. The difference between defect-free copper and that having a vacancy was shown to be quite modest for the [110] and [100] directions. It is very likely that other defects such as dislocations, grain boundaries, and dissolved gases could also alter the SEY characteristics. The presence of gas in the metal could lead to dynamic variations, with possible outgassing during the operation of such high power devices, leading to changes in the SEY characteristics. Such aspects can be studied through Molecular Dynamics simulations, and will be reported elsewhere. The present results also underscore the potential benefits of annealing or reducing surface roughness and inhomogeneities through laser or charged particle beam treatments.

\section{ACKNOWLEDGMENTS}

One of us (R.P.J.) acknowledges fruitful discussions with Maciej P. Polak and Dane Morgan (University of Wisconsin). This research was supported, in part, by a Department of Defense MURI under Grant No. FA9550-18-1-0062 on "Multipactor and Breakdown Susceptibility and Mitigation in Space-Based RF Systems" through a subaward from Michigan State University to Texas Tech University. The work at Texas Tech University was also supported through grants from the Office of Naval Research (ONR) (No. N00014-18-1-2382) and the Air Force Office of Scientific Research (No. FA9550-19-1-0056).

\section{REFERENCES}

${ }^{1}$ M. Izawa, Y. Sato, and T. Toyomasu, Phys. Rev. Lett. 74, 5044 (1995).

${ }^{2}$ K. Ohmi, Phys. Rev. Lett. 75, 1526 (1995).

3. R. M. Vaughan, IEEE Trans. Electron Devices 35, 1172 (1988).

${ }^{4} J$. Chang, P. Lawless, and T. Yamamoto, IEEE Trans. Plasma Sci. 19, 1152 (1991).

${ }^{5}$ J. R. M. Vaughan, IRE Trans. Electron Devices 8, 302 (1961).

${ }^{6}$ H. C. Kim and J. P. Verboncoeur, Phys. Plasmas 12, 123504 (2005).

${ }^{7}$ R. A. Kishek, Y. Y. Lau, L. K. Ang, A. Valfells, and R. M. Gilgenbach, Phys. Plasmas 5, 2120 (1988).

${ }^{8}$ N. Balcon, D. Payan, M. Belhaj, T. Tondu, and V. Inguimbert, IEEE Trans. Plasma Sci. 40, 282 (2012).

${ }^{9}$ A. S. Gilmore, Microwave Tubes (Artech House, Norwood, MA, 1986), p. 474.

${ }^{10}$ Y. Saito, S. Michizono, S. Anami, and S. Kobayashi, IEEE Trans. Electr. Insul. 28, 566 (1993).

${ }^{11}$ K. J. Kleman, in IEEE Proceedings of the 1993 Particle Accelerator Conference (IEEE, Piscataway, NJ, 1993), p. 924.

${ }^{12}$ E. G. Schweppe, R. Bachmor, and E. Demmel, in IEEE Proceedings of the 1993 Particle Accelerator Conference (IEEE, Piscataway, NJ, 1993), p. 1178.

${ }^{13}$ F. Hohn, W. Jacob, R. Beckmann, and R. Wilhelm, Phys. Plasmas 4, 940-944 (1997).

${ }^{14}$ N. Rozario, H. F. Lenzing, K. F. Reardon, M. S. Zarro, and C. G. Baran, IEEE Trans. Microw. Theory Tech. 42, 558 (1994).

${ }^{15}$ R. A. Kishek and Y. Y. Lau, Phys. Rev. Lett. 75, 1218 (1995).

${ }^{16}$ R. A. Kishek and Y. Y. Lau, Phys. Plasmas 3, 1481 (1996).

${ }^{17}$ V. P. Gopinath, J. P. Verboncoeur, and C. K. Birdsall, Phys. Plasmas 5, 1535 (1998).

${ }^{18}$ A. R. Martin, J. British Interplanetary Society 47, 134 (1994).

${ }^{19}$ C. Watts, M. Gilmore, and E. Schamiloglu, IEEE Trans. Plasma Sci. 39, 836 (2011).

${ }^{20}$ D. Cahen and A. Kahn, Adv. Mater. 15, 271 (2003).

${ }^{21}$ R. W. Strayer, W. Mackie, and L. W. Swanson, Surf. Sci. 34, 225 (1973).

${ }^{22}$ A. Kahn, Mater. Horizons 3, 7 (2016).

${ }^{23}$ V. E. Semenov, E. I. Rakova, D. Anderson, M. Lisak, and J. Puech, Phys. Plasmas 14, 033501 (2007).

${ }^{24}$ A. Iqbal, J. Verboncoeur, and P. Zhang, Phys. Plasmas 25, 043501 (2018).

${ }^{25}$ S. Anza, M. Mattes, C. Vicente, J. Gil, D. Raboso, V. E. Boria, and B. Gimeno, Phys. Plasmas 18, 032105 (2011).

${ }^{\mathbf{2 6}}$ S. Riyopoulos, Phys. Plasmas 5, 305 (1998).

${ }^{27}$ L. K. Ang, Y. Y. Lau, R. A. Kishek, and R. M. Gilgenbach, IEEE Trans. Plasma Sci. 26, 290 (1998).

${ }^{\mathbf{2 8}}$ A. Valfells, J. P. Verboncoeur, and Y. Y. Lau, IEEE Trans. Plasma Sci. 28, 529 (2000).

${ }^{29}$ G. Cheng, L. Liu, Y. Liu, and C. Yuan, IEEE Trans. Plasma Sci. 37, 1968 (2009).

${ }^{30}$ J. Vaughan, IEEE Trans. Electron Devices 36, 1963 (1989).

${ }^{31}$ C. Vicente, M. Mattes, D. Wolk, H. L. Hartnagel, J. R. Mosig, and D. Raboso, "Multipactor breakdown prediction in rectangular waveguide based components," in 2005 IEEE MTT-S International Microwave Symposium Digest (IEEE, New York, CA, 2005), Vol. 2, pp. 1055-1058.

${ }^{32}$ M. A. Furman and M. T. F. Pivi, Phys. Rev. Spec. Top. 5, 124404 (2002).

${ }^{33}$ D. R. Penn, Phys. Rev. B 35, 482 (1987).

${ }^{34}$ E. M. Baroody, Phys. Rev. 78, 780 (1950).

${ }^{35}$ M. Dapor, J. Electron Spectrosc. Relat. Phenom. 151, 182 (2006).

${ }^{36}$ D. C. Joy, J. Microsc. 147, 51 (1987).

${ }^{37}$ R. Shimizu and Z. J. Ding, Rep. Prog. Phys. 55, 487 (1992).

${ }^{38}$ K. Murata, D. F. Kyser, and C. H. Ting, J. Appl. Phys. 51, 4396 (1981).

${ }^{39}$ Z. J. Ding and R. Shimizu, Scanning 18, 92 (1996).

${ }^{40} \mathrm{~S}$. C. Luo and D. C. Joy, "Fundamental electron and ion beam interactions with solids for microscopy, microanalysis and microlithography," in Scanning Microscopy (Suppl 4), edited by J. Schou, P. Kruit, and D. E. Newbury (Scanning Microscopy International, Chicago, 1990), pp. 127-146. 
${ }^{41}$ D. E. Newbury, R. L. Myklebust, and E. B. Steel, in Microbeam Analysis1990, edited by J. R. Michael and P. Ingram (San Fransico Press, San Francisco, 1990), pp. 127-130.

${ }^{42} \mathrm{M}$. Dapor, Nucl. Instrum. Methods Phys. Res. B 269, 1668 (2011).

${ }^{43}$ D. C. Joy, Scanning Microsc. 5, 329 (1991).

${ }^{44}$ D. C. Joy and S. Luo, Scanning 11, 176 (1989).

${ }^{45}$ H. Bethe, Ann. Phys. 5, 325 (1930).

${ }^{46} \mathrm{H}$. A. Bethe and J. Ashkin, Exp. Nucl. Phys. 1, 166 (1953).

${ }^{47}$ R. M. Nieminen, Scanning Microsc. 2, 1917 (1988).

${ }^{48}$ Z. J. Ding, X. D. Tang, and R. Shimizu, J. Appl. Phys. 89, 718 (2001).

${ }^{49}$ Z. J. Ding and R. Shimizu, Surf. Sci. 222, 313 (1989).

${ }^{\mathbf{5 0}}$ N. F. Mott and H. S. W. Massey, The Theory of Atomic Collisions, 3rd ed. (Oxford University Press, 1965).

${ }^{51}$ P. Hohenberg and W. Kohn, Phys. Rev. 136, B864 (1964).

${ }^{52}$ Y. He and T. Zeng, J. Phys. Chem. C 114, 18023 (2010).

${ }^{53}$ K. Glantschnig and C. Ambrosch-Draxl, New J. Phys. 12, 103048 (2010).

${ }^{54}$ J. Yan, K. W. Jacobsen, and K. Y. Thygesen, Phys. Rev. B 84, 235430 (2011).

${ }^{\mathbf{5 5}} \mathrm{See}$ http://cms.mpi.univie.ac.at/vasp for more information about the VASP simulator.

${ }^{\mathbf{5 6}}$ G. Kresse and J. Hafner, Phys. Rev. B 47, 558 (1993).

${ }^{57} \mathrm{G}$. Kresse and J. Furthmuller, Phys. Rev. B 54, 11169 (1996).

${ }^{58}$ D. Vanderbilt, Phys. Rev. B 41, 7892 (1990).

59. P. Perdew, K. Burke, and M. Ernzerhof, Phys. Rev. Lett. 77, 3865 (1996).

${ }^{60}$ P. E. Blöchl, Phys. Rev. B 50, 17953 (1994).

${ }^{61}$ G. Kresse and D. Joubert, Phys. Rev. B 59, 1758 (1999).

${ }^{62}$ H. J. Monkhorst and J. D. Pack, Phys. Rev. B 13, 5188 (1976).

${ }^{63}$ M. Gajdoš, K. Hummer, G. Kresse, J. Furthmüller, and F. Bechstedt, Phys. Rev. B 73, 045112 (2006).

${ }^{64}$ M. Sanati, R. C. Albers, T. Lookman, and A. Saxena, Phys. Rev. B 88, 024110 (2013).

${ }^{65}$ O. Alsalmi, M. Sanati, R. C. Albers, T. Lookman, and A. Saxena, Phys. Rev. M 2, 113601 (2018).

66. Nagy, A. Arnau, and P. M. Echenique, Phys. Rev. A 40, 987 (1989).

${ }^{67}$ F. Yubero, J. M. Sanz, B. Ramskov, and S. Tougaard, Phys. Rev. B 53, 9719 (1996).

${ }^{68} \mathrm{M}$. Polak, University of Wisconsin, private communication (2019).
${ }^{69}$ A. B. Pippard, "Ultrasonic attenuation in metals," Philos. Mag. 46, 1104 (1955).

${ }^{70}$ H. K. A. Nguyen, J. Mankowski, J. Dickens, A. Neuber, and R. P. Joshi, IEEE Trans. Plasma Sci. 47, 1364 (2019).

${ }^{71}$ H. K. A. Nguyen, J. Mankowski, J. C. Dickens, A. Neuber, and R. P. Joshi, AIP Adv. 8, 015325 (2018).

${ }^{72}$ H. Qiu, S. Prasad, L. Ludeking, R. P. Joshi, and E. Schamiloglu, J. Appl. Phys. 115, 193303 (2014)

${ }^{73}$ H. M. Polatoglou, M. Methfessel, and M. Scheffle, Phys. Rev. B 48, 1877 (1993).

${ }^{74}$ P. O. Gartland, S. Berge, and B. J. Slagsvold, Phys. Rev. Lett. 28, 738 (1972).

${ }^{75}$ V. Baglin, J. Bojko, O. Gröbner, B. Henrist, N. Hilleret, C. Scheuerlein, and M. Taborelli, "The secondary electron yield of technical materials and its variation with surface treatments," in Proceedings of EPAC, Vienna, Austria (CERN, 2000), pp. 217-221.

${ }^{76}$ Z. J. Ding, H. M. Li, X. D. Tang, and R. Shimizu, Appl. Phys. A 78, 585 (2004).

${ }^{77}$ H. B. Zhang, X. C. Hu, R. Wang, M. Cao, N. Zhang, and W. Cui, Rev. Sci. Instrum. 83, 066105 (2012).

${ }^{78}$ F. Le Pimpec, R. E. Kirby, F. K. King, and M. Pivi, Nucl. Instrum. Methods Phys. Res. A 564, 44 (2006).

${ }^{79}$ J. Yang, W. Z. Cui, Y. Li, G. B. Xie, N. Zhang, and R. Wang, Appl. Surf. Sci. 382, 88 (2016).

${ }^{80}$ X. C. Hu, M. Cao, and W. Z. Cui, Micron 90, 71 (2016).

${ }^{81}$ R. M. Stern and H. Taub, Phys. Rev. Lett. 20, 1340 (1968).

${ }^{82}$ M. Ye, Y. N. He, S. G. Hu, J. Yang, R. Wang, T. C. Hu, W. B. Peng, and W. Z. Cui, J. Appl. Phys. 114, 104905 (2013).

${ }^{83}$ M. Pivi, F. K. King, R. E. Kirby, T. O. Raubenheimer, G. Stupakov, and F. Le Pimpec, J. Appl. Phys. 104, 104904 (2008).

${ }^{84}$ H. E. Farnsworth, Phys. Rev. 34, 679 (1929).

${ }^{85} \mathrm{M}$. Ye, Y. N. He, S. G. Hu, R. Wang, T. C. Hu, J. Yang, and W. Z. Cui, J. Appl. Phys. 113, 074904 (2013).

${ }^{86}$ C. Swanson and I. D. Kaganovich, J. Appl. Phys. 120, 213302 (2016).

${ }^{87}$ M. Q. Ding, M. G. Huang, J. J. Feng, G. D. Bai, and T. C. Yan, Appl. Surf. Sci. 255, 2196 (2008).

${ }^{88}$ J. Luo, P. Tian, C. T. Pan, A. W. Robertson, J. H. Warner, E. W. Hill, and G. A. D. Briggs, ACS Nano 5, 1047 (2011). 